\title{
Neuropsicologia e Oncologia Pediátrica: um diálogo em emergência
}

\author{
Danielle Garcia \\ Ediana Rosselly de Oliveira Gomes \\ Bruna Balaban Garcia \\ Débora Sunaly Leite da Silva \\ Izabel Hazin* \\ Universidade Federal do Rio Grande do Norte, Natal, Brasil
}

\begin{abstract}
RESUMO
Dados do INCA estimam que anualmente cerca de 11.530 casos de neoplasias são diagnosticados em crianças e adolescentes. $\mathrm{O}$ aumento do número de sobreviventes vem crescendo, junto com as sequelas cognitivas decorrentes da doença e de seu tratamento. Nesse sentido, o presente artigo discute acerca da pertinência do estabelecimento de diálogo entre a neuropsicologia e a oncologia. $\mathrm{O}$ artigo aborda os dois subtipos de câncer mais comuns na infância e adolescência: os tumores de fossa posterior e a leucemia linfoide aguda. A discussão será ilustrada com resultados oriundos de dois estudos distintos realizados em serviços públicos de referência no tratamento do câncer pediátrico na Região Nordeste do Brasil.
\end{abstract}

Palavras-chave: neuropsicologia; oncologia; desenvolvimento.

\begin{abstract}
Neuropsychology and Oncology: an emerging dialogue

INCA data estimate that around 11.530 cases of neoplasias are diagnosed yearly among children and teenagers. On the other hand, the number of survivors of these diseases has been increasing, together with cognitive sequels related to the illness and its treatment. In this context, the present paper discusses the need of establishing a dialogue between neuropsychology and oncology. Two of the most common types of cancer occurring during childhood and adolescence are addressed: tumors of posterior fossa and acute lymphoblastic leukemia. Data issued from two empirical studies developed in North-East Brazilian region public health services for pediatric cancer treatment will illustrate the discussion mentioned above.
\end{abstract}

Keywords: neuropsychology; oncology; development.

\section{INTRODUÇÃO}

Dados do Instituto Nacional de Câncer (2012) estimam que a incidência dos tumores pediátricos no mundo varie de $1 \%$ a $3 \%$ do total de casos de câncer. No Brasil, o percentual mediano dos tumores pediátricos observados nos Registros de Câncer de Base Populacional (RCBP) encontra-se próximo de $3 \%$. Sendo assim, a expectativa é que ocorrerão anualmente cerca de 11.530 novos casos de neoplasias em crian- ças e adolescentes. Tal estimativa prevê aumento na incidência dos tumores pediátricos quando comparado ao total estimado no biênio 2010-2011, que foi de 9.386 novos casos.

As últimas informações disponíveis acerca da taxa de mortalidade brasileira mostram que no ano de 2009 os óbitos por neoplasias para a faixa etária de 1 a 19 anos encontraram-se entre as dez primeiras causas de morte (INCA, 2012). No entanto, constata-se o au- 
mento significativo da sobrevida, decorrente do diagnóstico precoce e da eficácia dos tratamentos antineoplásicos. É preciso salientar que o aumento do número de crianças e adolescentes sobreviventes do câncer, notadamente os tumores do Sistema Nervoso Central (SNC) e as Leucemias, vem acompanhado da emergência de sequelas cognitivas e motoras, decorrentes da doença e de seus tratamentos, impactando diretamente a qualidade de vida desta população clínica.

Os déficits cognitivos são comumente identificados dentre os sobreviventes de câncer. Embora as disfunções cognitivas sejam mais reportadas durante as fases ativas do tratamento, déficits específicos são notados em todas as etapas do adoecimento, e mesmo anos após o término do tratamento (Noogle \& Dean, 2013). Os insultos neurológicos na infância têm expressão clínica bastante distinta dos que ocorrem em idade adulta, uma vez que, no cérebro infantil, as etapas maturacionais ainda estão em curso, a especialização hemisférica ainda não se encontra consolidada e, portanto, a topografia funcional do cérebro ainda não se encontra plenamente estabelecida (Muszkat, 2008).

Neste desafio, a abordagem neuropsicológica ganha destaque, pois esta possibilita a apreciação do potencial de interferência de diferentes fatores, isolados e em interação. Os dados oriundos da avaliação em neuropsicologia permitem o mapeamento de áreas cognitivas preservadas e deficitárias. Adicionalmente, avança na investigação do impacto destas sobre a aprendizagem, as atividades de vida diária e as relações socioafetivas dos sobreviventes. Por fim, tais resultados oferecem subsídios para a proposição de estratégias de intervenção eficazes, que garantam o potencial de desenvolvimento e aprendizagem deste grupo clínico.

$\mathrm{Na}$ abordagem neuropsicológica do câncer infantil é preciso considerar dois aspectos centrais. $\mathrm{O}$ primeiro aspecto está associado à imaturidade do cérebro infantil, o que o torna mais vulnerável aos agentes neurotóxicos associados à quimioterapia (Duffner, 2010). Em segundo lugar, merece destaque, notadamente para os tumores de SNC, a constatação que a incidência de um evento lesional na infância implica na sobreposição dos processos naturais de maturação por mecanismos de reorganização, podendo levar a importantes prejuízos no desenvolvimento dos substratos cognitivos das funções neuropsicológicas mais complexas, levando a alterações no desenvolvimento da estrutura funcional cerebral e a um funcionamento cognitivo qualitativamente diferente (Mello et al., 2006).

Diante do exposto, circunscreve-se neste estudo a pertinência do estabelecimento de diálogo entre a neuropsicologia infantil e a oncologia pediátrica, em especial em termos de dois subtipos específicos do câncer, a saber, os tumores de fossa posterior (TFP) e a leucemia linfoide aguda (LLA). Tal delimitação aqui executada é justificada pela alta incidência destes tipos de câncer na infância, bem como a implicação direta destas neoplasias e seus tratamentos sobre a organização e o funcionamento do Sistema Nervoso Central.

Para tanto, o presente artigo problematiza acerca destas duas modalidades de neoplasias pediátricas, caracterizando seus processos de adoecimento, seus tratamentos, bem como as implicações destes para o funcionamento neuropsicológico de crianças e adolescentes sobreviventes. Apresentará resultados oriundos de dois estudos distintos realizados em serviços públicos de referência no tratamento do câncer pediátrico na Região Nordeste do Brasil.

\section{TUMORES DE FOSSA POSTERIOR}

Os tumores de Sistema Nervoso Central - SNC são, na maioria das populações, o segundo tipo mais frequente de câncer na infância, superados apenas pelas leucemias (Ribeiro \& Latorre, 2003). Dentre as neoplasias sólidas, no entanto, tais tumores são os mais frequentes na faixa pediátrica, correspondendo a $20 \%$ de todas as doenças malignas na infância (Reeves et al., 2006).

Em crianças, os tumores de SNC têm características peculiares, sendo mais comuns lesões infratentoriais - ou de fossa posterior - nomenclatura utilizada para designar a região localizada abaixo do tentório ou tenda do cerebelo, membrana de dura-máter que recobre esta estrutura e sustenta os lobos occipitais do cérebro (Furrer \& Suzuki, 2003). Na fossa posterior, dentre os tipos de tumores mais comuns encontram-se os meduloblastomas e os gliomas de baixo grau, dentre os quais se destacam os astrocitomas pilocíticos (Mabbott, Penkman, Witol \& Strother, 2008; Vaquero et al., 2008). Estes subtipos de tumores serão objeto de investigação do presente estudo. 
Os astrocitomas são tumores de origem neuroepitelial, derivados de células astrocíticas da neuroglia. Correspondem a aproximadamente um terço dos tumores de SNC na infância, localizando-se mais frequentemente nos hemisférios cerebelares. A maior incidência ocorre na primeira década de vida, com idade média entre 6,5 e nove anos, sem predomínio de gênero. Geralmente está associado a curso clínico relativamente benigno devido, sobretudo, à lentidão mitótica de suas células e à sua natureza histológica discreta e bem circunscrita - fatores que favorecem a ressecção cirúrgica completa e a isenção de tratamentos antineoplásicos complementares (Rondinelli, 2003).

O meduloblastoma é um tumor embrionário maligno de linhagem neuroectodérmica, que se origina predominantemente na área do vermis cerebelar (Palmer et al., 2007), correspondendo entre 10 a 20\% dos casos de tumores de SNC em crianças (Furrer \& Suzuki, 2003). Sua ocorrência é mais frequente na primeira década de vida, sendo a idade média de ocorrência 7,3 anos, com picos de incidência aos três e sete anos de idade, em proporção masculino/feminino variando de 1,1:1 a 2,6:1 (Gottardo e Gajjar, 2006; Furrer \& Suzuki, 2003). É um tumor fortemente maligno, sua histologia é caracterizada por alto grau de celularidade e potencial mitótico, grande potencial de infiltração e forte propensão à disseminação através do líquor e ao longo do neuro-eixo, caracterizando piores prognósticos. Devido ao alto grau de malignidade e aos riscos de recidivas, requer intervenções terapêuticas agressivas. Além da cirurgia de ressecção, são realizadas intervenções complementares, como quimioterapia e radioterapia crânioespinhal acrescida de reforço na fossa posterior.

\section{Cirurgia}

A cirurgia é considerada uma estratégia eficiente e rápida para redução da multiplicação de células neoplásicas, sendo assim, é a primeira abordagem terapêutica utilizada no tratamento de tumores sólidos, como os tumores de FP. Diversos estudos, contudo, têm indicado que o procedimento cirúrgico para a ressecção de tumores localizados na região da fossa posterior (FP) pode ocasionar comprometimentos em estruturas cerebrais, com destaque para lesões cerebelares. Alguns trabalhos têm feito alusão ao papel desempenhado pelo cerebelo em funções cognitivas não motoras, tendo sido observados prejuízos neuropsico- lógicos de caráter pré-frontal em pacientes submetidos à ressecção cirúrgica de lesões cerebelares benignas. Estes estudos sugerem que as conexões entre o cerebelo e o córtex pré-frontal dorsolateral são prejudicadas por tumores de FP e pelo procedimento cirúrgico invasivo, interferindo, assim, no desenvolvimento das funções executivas, da memória operacional e da atenção (Budisavlyevic \& Rammani, 2012; Law, Bouffet \& Laughlin, 2011; Stargatt, Anderson \& Rosenfeld, 2002; Riva \& Giorgi, 2000; Varela, Liakopoulou \& Alexiou, 2011).

\section{Quimioterapia e Radioterapia para os Tumores de FP}

A radioterapia é incluída em muitos protocolos terapêuticos para tumores de FP com o objetivo de diminuir as chances de recidiva tumorais locais e a distância, contudo essa modalidade terapêutica tem demonstrado impacto adverso sobre o neurodesenvolvimento, sendo responsável por severos déficits neurocognitivos, especialmente em crianças na primeira infância. Os efeitos cognitivos relacionados à radioterapia abrangem o funcionamento intelectivo, o desempenho acadêmico, a memória, a atenção e a velocidade de processamento. Esses sintomas têm sido referidos como progressivos, aumentando com o tempo decorrido após o tratamento. Acredita-se que a relação entre a radiação craniana e o declínio neurocognitivo observado ocorre devido a danos à substância branca cortical e subcortical - que apresenta maior vulnerabilidade durante a infância (Palmer, Reddick \& Gajjar, 2007; Moore, 2005; Mulhern et al., 1999; Maddrey et al., 2005).

Nos protocolos de tratamento atuais para tumores de FP tem-se incluído drogas quimioterápicas com vistas à diminuição ou suspensão das doses de radioterapia. Especial importância tem sido atribuída ao uso da quimioterapia no tratamento de crianças com baixa idade ao diagnóstico, situação em que são administradas doses altas de quimioterápicos com vistas ao controle adequado da doença sem o recurso à radioterapia ou retardando o seu uso. Contudo, alguns trabalhos indicam que a associação entre a radioterapia e a quimioterapia pode resultar em danos ainda mais severos ao sistema nervoso em desenvolvimento (Massimino, Cefalo \& Riva, 2012; Brinkman, Reddick \& Luxton 2012; Watanabe, Azami \& Ozawa, 2011). 
Há controvérsias na literatura acerca dos efeitos cognitivos da quimioterapia quando administrada isoladamente. De um modo geral, alguns consensos já podem ser delineados a respeito das repercussões da quimioterapia no desenvolvimento, dentre eles, destacam-se: dificuldades no processamento visual, no funcionamento viso-motor, na atenção e no funcionamento executivo. Esse padrão de funcionamento tem sido referido como semelhante aos achados em crianças tratadas com radioterapia, diferindo apenas em intensidade e severidade (Massimino, Cefalo, \& Riva, 2012; Anderson \& Kunin-Batson, 2009).

\section{Estudo llustrativo 1: Tumores de Fossa Posterior}

O estudo de Garcia (2011) investigou os efeitos dos tumores de fossa posterior e seu tratamento sobre a capacidade intelectiva de 21 crianças brasileiras com idades entre sete e 15 anos diagnosticadas com astrocitoma (13) e meduloblastoma (8), atendidas no Instituto de Medicina Integral Professor Fernando Figueira - IMIP/Centro de Hematologia e Oncologia Pediátrica - CEHOPE, em Recife/PE entre os anos 2003 a 2010. A capacidade intelectiva das crianças foi avaliada através das Escalas Wechsler de Inteligência para Crianças - WISC-III, e os dados foram submetidos à análise estatística através de ferramenta inferencial não-paramétrica (teste Ü de Mann-Whitney) - com nível de significância de 0,05 para rejeição da hipótese nula, a partir da investigação do impacto das seguintes variáveis: i) diagnóstico e tipo de tratamento ao qual a criança foi submetida; ii) idade da criança ao diagnóstico; iii) tempo decorrido entre o diagnóstico e a avaliação da capacidade intelectiva e; iv) nível de escolaridade da mãe. Estas variáveis foram analisadas na amostra total de crianças participantes, bem como no interior dos dois grupos de diagnóstico.

A variável diagnóstico/tratamento foi responsável por diferenças estatisticamente significativas entre os desempenhos das crianças no QI de Execução - QIE, no Índice Fatorial Velocidade de Processamento IVP, tendo as crianças com meduloblastoma apresentado desempenhos significativamente abaixo do esperado nestes domínios. Uma vez que os contrastes mais evidentes se situaram no IVP, acredita-se que este índice tenha contribuído de forma decisiva para o baixo desempenho das crianças com meduloblastoma na escala de execução, apontando para prejuízos no âmbito da velocidade psicomotora e mental no processamento de informações.

No que concerne à idade da criança quando diagnosticada e tratada, o desempenho das crianças com meduloblastoma não apresentou contrastes estatisticamente significativos em função da idade da criança ao diagnóstico. A não diferenciação entre os grupos etários no interior do grupo de crianças com meduloblastoma pode ser atribuída aos seguintes fatores: a) a forte expressão de variáveis intervenientes neste subgrupo, como a alto grau de malignidade do tumor, complicações associadas - como a hidrocefalia, o baixo nível de escolaridade dos pais e a agressividade do tratamento, cujos efeitos podem ter ocultado o impacto da idade ao diagnóstico e; b) baixo efetivo de participantes, que impediu o estabelecimento de conclusões mais efetivas, bem como impossibilitou a verificação dos efeitos de interação destas variáveis. São necessários estudos adicionais, com maior efetivo de participantes, visando verificar tal influência sobre o desempenho cognitivo de crianças diagnosticadas com meduloblastoma.

Dentre as crianças com astrocitoma, entretanto, houve contrastes estatisticamente significativos no QI Verbal (QIV), e no Índice Fatorial Compreensão Verbal (ICV) em favor das crianças diagnosticadas precocemente. Este resultado sugere que as crianças de G1 diagnosticadas precocemente (idade $\leq 6 \mathrm{anos}$ ) apresentam maior preservação das funções de linguagem, reforçando o papel do cerebelo neste domínio. Uma vez que é sabido que o cerebelo apresenta grande potencial de reorganização e que os hemisférios cerebrais apresentam maior equipotencialidade em etapas precoces do neurodesenvolvimento, justifica-se o menor prejuízo cognitivo entre crianças diagnosticadas mais cedo. O diagnóstico precoce, assim, seria fator de proteção ante o surgimento de danos cognitivos pós-tratamento de tumores cerebelares benignos.

No que concerne à variável tempo decorrido entre o diagnóstico e a avaliação da capacidade intelectiva, observou-se que dentre as crianças com astrocitoma, não houve contrastes estatisticamente significativos. Este dado pode ser explicado pelo fato de que a cirurgia de ressecção de tumores cerebelares benignos acarreta efeitos neurocognitivos focais e agudos, causando déficits cognitivos imediatos, aos quais se se- 
guem prontos mecanismos de reorganização do SNC, que não sofre alterações significativas ao longo do tempo.

No grupo de crianças diagnosticadas com meduloblastoma houve contrastes estatisticamente significativos no desempenho do QI de Execução, QI Total, Índice Fatorial Resistência à Distração e Índice Fatorial Velocidade de Processamento, em favor das crianças com menos tempo entre diagnóstico e tratamento, dado que sugere declínio tardio e progressivo da capacidade intelectiva, notadamente no domínio nãoverbal. Este resultado é condizente com os diversos estudos que apontam que crianças submetidas à radioterapia de crânio e neuro-eixo apresentam declínios na capacidade intelectiva, resultantes da perda progressiva de substância branca, da subsequente lentidão no processamento de informações e de falhas na aquisição de habilidades cognitivas em ritmo comparável ao de crianças de mesma idade e escolaridade.

No que diz respeito à variável escolaridade da mãe, foram encontrados contrastes estatisticamente significativos na amostra total de crianças participantes, e no grupo de crianças com astrocitoma. Tais resultados encontram relação com estudos que apontam relação significativa entre variáveis socioculturais e o desenvolvimento cognitivo, reforçando a influência destas sobre a capacidade intelectiva de crianças em condições normais ou patológicas. O baixo nível educacional materno (e suas consequências socioeconômicas) confere defasagem prévia ao desenvolvimento cognitivo da criança, colocando-a em potencial desvantagem no que concerne às possibilidades de estimulação ambiental na direção de uma melhor reorganização do SNC.

\section{LEUCEMIA LINFOIDE AGUDA}

As leucemias são doenças clonais originárias da transformação neoplásica de células progenitoras hematopoéticas da medula óssea, que adquirem alterações genéticas que lhes conferem uma vantagem proliferativa e de sobrevivência ou um prejuízo da diferenciação. Esse clone neoplásico prolifera-se até substituir o parênquima medular normal, com prejuízo da hematopoese e posterior disseminação pelo organismo (Pui, Carroll, Meshinchi \& Arceci, 2011).

As leucemias são classificadas a partir de dois aspectos: o tipo de células afetadas e a velocidade do desenvolvimento e progressão da doença. Da combinação destas duas categorias de classificação decorrem os seguintes tipos da doença: a) Leucemia Mieloide Aguda; b) Leucemia Mieloide Crônica; c) Leucemia Linfoide Crônica e; d) Leucemia Linfoide Agu$d a$, caso específico abordado por este estudo.

O tratamento da LLA é bastante extenso, variando em média de 2 a 3 anos, o que pode acarretar graves consequências para a vida social e acadêmica dos pacientes. (Oliveira et al., 2004; Pedrosa \& Lins, 2002). Apesar de existir variações entres os esquemas terapêuticos utilizados, há relativa homogeneidade em termos das fases que atualmente constituem os protocolos, evidenciando-se geralmente as etapas abaixo (Filho, Jr, Cristofani, Almeida \& Teixeira, 2012):

1. Indução da Remissão: nesta fase, o objetivo é eliminar mais de $99 \%$ das células leucêmicas e restaurar a hematopoese normal e o estado clínico do paciente. Em geral, essa fase inclui o uso de glicocorticoides (dexametasona, prednisona), vincristina e pelo menos um terceiro agente, como asparaginase e/ou antraciclina.

2. Intensificação e Consolidação Tardia: esta fase se inicia quando a hematopoese é restaurada e contribui para a melhora da sobrevida livre de eventos. Metotrexato em altas doses e 6-mercaptopurina, altas doses de asparaginase e esquemas semelhantes à indução são comumente utilizados.

3. Manutenção: após intensificação, os pacientes com LLA devem receber terapia de manutenção por 18 e 30 meses, geralmente constituída da combinação de 6-mercaptopurina e metotrexato, administrados à máxima tolerância.

4. Transplante Alogênico de Medula Óssea: a maioria dos estudos dessa modalidade terapêutica é feita em pacientes em segunda remissão da doença e com resultados ruins.

5. Tratamento do Sistema Nervoso Central (SNC): A ausência de terapia específica para o SNC resulta em até $75 \%$ de recaídas nesse local. Com a introdução de tratamento profilático específico para o SNC com quimioterapia intratecal e radioterapia craniana combinadas, o número de recaídas isoladas em SNC foi reduzido a cerca de $5 \%$. 


\section{Quimioterapia e radioterapia para a LLA}

No tratamento da LLA a radiação craniana e os agentes quimioterápicos têm sido administrados na fase do tratamento destinada à prevenção da doença no Sistema Nervoso Central (SNC). Assim como se observa no tratamento para tumores de FP, os estudos acerca da repercussão da quimioterapia e radioterapia no funcionamento cognitivo pós tratamento para a LLA têm indicado que estas modalidades terapêuticas, por serem potencialmente neurotóxicas, produzem efeitos deletérios, persistentes e permanentes no funcionamento neurocognitivo, os quais com freqüência são evidenciados tardiamente (Cole \& Kamen, 2006).

A quimioterapia intratecal tripla isolada, substituindo a irradiação craniana, tem sido considerada o tratamento padrão para crianças diagnosticadas com LLA. No entanto, essa abordagem terapêutica também tem sido associada a déficits neurocognitivos significativos, em especial nos domínios da atenção, velocidade de processamento de informação, funcionamento executivo, habilidade motora fina e na memória verbal (Campbell et al., 2007; Peterson et al., 2008).

Os estudos que avaliam o desempenho neurocognitivo de crianças com LLA submetidas apenas à quimioterapia são controversos. Alguns trabalhos indicam que as crianças submetidas a este protocolo apresentam um bom desempenho neurocognitivo a longo prazo, todavia, recentemente têm-se feito alusão a efeitos tardios ocasionados pela quimioterapia profilática em funções cognitivas específicas, como a atenção, velocidade de procesamento, memória, compreensão verbal, habilidades visuo-espaciais e funcionamento visuo-motor (Temming \& Jenney 2011).

De acordo com Moleski (2000), embora não haja consenso sobre a gama prejuízos a longo prazo ocasionado pela radioterapia e quimioterapia em sobreviventes de LLA, pode-se afirmar com segurança que aqueles que receberam uma combinação de radioterapia e metotrexato intratecal apresentam déficits na memória de longo prazo, memória visoespacial, memória verbal, memória auditiva, na metacognição, reconhecimento verbal, em habilidades visoespaciais e motoras, na velocidade de processamento e atenção.

\section{Estudo ilustrativo 2: Leucemia Linfoide Aguda}

O estudo realizado por Gomes (2011) investigou o perfil neuropsicológico de pacientes pediátricos diagnosticados com leucemia linfoide aguda, atendidos pela Liga Norte Riograndense Contra o Câncer, Hospital Infantil Varela Santiago, Casa Durval Paiva de Apoio à Criança com Câncer e o Grupo de Apoio à Criança com Câncer, instituições situadas em Natal/RN. Participaram do estudo 20 (vinte) crianças, de ambos os sexos, com idades entre seis e doze anos, diagnosticadas com LLA e submetidas apenas à quimioterapia intratecal como profilaxia da doença no SNC. Destas, 10 (dez) encontravam-se em tratamento e 10 (dez) estavam fora de tratamento há pelo menos 1 (um) ano; 14 (catorze) crianças do sexo feminino e 06 (seis) do sexo masculino; 09 (nove) iniciaram o tratamento antes dos cinco anos de idade, enquanto 11 (onze) crianças tinham cinco anos ou mais quando do diagnóstico.

O estudo foi delineado observando-se as variáveis relevantes levantadas pela literatura da área, no que concerne aos efeitos cognitivos relacionados aos impactos do tratamento profilático da LLA, quais sejam, sexo da criança, idade no momento do diagnóstico e tempo decorrido desde o início do tratamento. Para análise desta última variável as crianças foram divididas nos grupos 1) em tratamento e 2) fora de tratamento há pelo menos 1 ano (Moleski, 2000). Para realização do estudo foram avaliados os seguintes domínios: capacidade intelectiva, sistemas atencionais, sistemas mnemônicos e funções executivas.

\section{Capacidade intelectiva}

A capacidade intelectiva foi avaliada a partir do WISC-III e do Teste Matrizes Progressivas de Raven. Os resultados indicaram melhor desempenho das crianças em tratamento em todas as medidas propostas pelos instrumentos. Observou-se melhor desempenho das crianças do sexo masculino em relação àquelas do sexo feminino em todos os QIs e Índices Fatoriais do WISC-III. No grupo que se encontrava fora de tratamento, crianças com menos de cinco anos ao diagnóstico alcançaram desempenho inferior, mesmo em medidas frequentemente preservadas nos demais grupos, tais como QI de Execução e Índice de Organização Perceptual, este subgrupo apresentou desempenho médio significativamente inferior. Tais resultados 
reforçam a hipótese de maior vulnerabilidade das estruturas cerebrais em processo de maturação diante dos insultos neurotóxicos do tratamento quimioterápico (Buizer et al., 2005).

Foram encontradas as seguintes diferenças estatisticamente significativas: a) nas medidas de QIV $(\mathrm{p}=0,031)$, QIE $(\mathrm{p}=0,037)$ e QIT $(\mathrm{p}=0,012)$ com melhor desempenho do sexo masculino em relação ao feminino; b) no grupo em tratamento, crianças com idade inferior aos 5 anos obtiveram melhor desempenho nos itens QIV ( $\mathrm{p}=0,029)$, QIE $(\mathrm{p}=0,05)$, QIT $(\mathrm{p}=0,011)$ ICV $(\mathrm{p}=0,033)$ e IRD $(\mathrm{p}=0,015) ; \mathrm{c})$ no grupo fora de tratamento, com diferenças significativas apenas para QIE $(p=0,015)$ e IOP $(p=0,025)$, indicando rendimento inferior para o subgrupo diagnosticado com menos de 5 anos de idade.

$\mathrm{O}$ quociente de inteligência global (QIT) fornecido pelo WISC-III foi menor que 90 (limiar inferior à média esperada) em $50 \%$ dos sujeitos avaliados. Tais pontuações rebaixadas situam-se principalmente nos grupos de risco associados aos impactos cognitivos do tratamento profilático da LLA. Destaca-se que o Índice Resistência à Distração foi classificado como inferior em praticamente todos os grupos e subgrupos analisados.

\section{Sistemas atencionais}

Os sistemas atencionais de seletividade e alternância foram avaliados a partir do Teste de Atenção por Cancelamento (Montiel \& Capovilla, 2007). A análise dos dados resultantes do estudo não revelou significância estatística entre os grupos e subgrupos propostos. Destaca-se, a identificação de escores mais rebaixados, nas partes 2 e 3 do teste. Estes dados reforçam a ideia de que crianças com LLA tratadas apenas com quimioterapia alcançam desempenho semelhante ao de crianças saudáveis nas atividades de atenção e processamento de informação, exceto no que concerne à flexibilidade atencional, contradizendo a queixa frequente de que crianças curadas da LLA vivenciam problemas relacionado à capacidade de atenção (Buizer et al., 2005 e Mennes et al., 2005).

\section{Funções executivas}

O funcionamento executivo foi avaliado através da aplicação do Teste Wisconsin de Classificação de Cartas. Os resultados evidenciaram que os grupos $\mathrm{em}$ tratamento e fora de tratamento, sexo masculino e feminino, obtiveram desempenho homogêneo e inferior ao esperado para os itens RP (respostas perseverativas), EP (erros perseverativos) e AA (aprendendo a aprender). No grupo em tratamento observou-se melhor desempenho do subgrupo diagnosticado antes dos 5 anos de idade. Já no grupo fora de tratamento, os dados revelaram melhor desempenho no grupo diagnosticado com idade igual ou superior a 5 anos. A análise estatística revela diferença significativa apenas no item Aprendendo a Aprender (AA) no grupo de crianças fora de tratamento, indicando desempenho inferior para o grupo diagnosticado com menos de cinco anos de idade $(\mathrm{p}=0,022)$.

Os itens destinados a avaliar o comportamento perseverativo forneceram os resultados mais rebaixados no instrumento, sendo classificados como moderadamente comprometidos, leve a moderadamente comprometidos e levemente comprometidos, sugerindo dificuldades na inibição de respostas alternativas ou inadequadas, atualização e monitoramento da informação e mudança de padrões mentais (Mello, 2008).

Estudos desenvolvidos na tentativa de compreender como o tratamento quimoterápico pode intervir sobre o funcionamento das áreas frontais do cérebro associadas às funções executivas e marcadas por um curso neurodesenvolvimental mais prolongado - identificam impactos na distribuição e proliferação da substância branca, e, portanto, mielinização das áreas frontais, como um dos principais fatores associados a tais déficits (Campbell et al., 2009; Carey et al., 2007; Moleski, 2000).

\section{Sistema mnemônico}

A avaliação do sistema mnemônico foi realizada a partir da aplicação dos instrumentos Recordação de História (RH) e Lista de Palavras (RAVLT), utilizados na investigação da memória episódica e semântica, respectivamente, nas modalidades imediata (I), como tardia (T). Os dados demonstram que o grupo fora de tratamento apresentou melhores resultados que o grupo em tratamento. No que concerne à investigação do funcionamento mnemônico das crianças a partir da variável sexo não se observou diferenças relevantes. Observou-se melhores resultados no grupo em tratamento para as crianças diagnosticadas antes dos 5 anos de idade. No grupo fora de tratamento, crianças diagnosticadas com cinco anos de idade ou 
mais alcançaram melhores resultados. De modo geral, a avaliação da memória episódica e semântica apresentaram-se dentro do esperado.

A memória operacional foi avaliada pelos subtestes Dígitos e Aritmética, do WISC-III, que combinados formam o Índice de Resistência à Distração. Houve rebaixamento importante da pontuação deste índice em decorrência, principalmente, do desempenho dos participantes no subteste de aritmética, que envolve outros recursos cognitivos além da memória operacional. A análise clínica da aplicação deste subteste revela dois aspectos principais relacionados a tais escores, quais sejam: a) a solicitação recorrente para que o examinador repetisse o problema apresentado; b) a dificuldade em realizar operações mentais sem auxílio de objetos concretos. A ausência de atividades capazes de avaliar outros domínios de forma específica, como conhecimento matemático, dificulta a elaboração de conclusões sobre tais achados.

\section{CONSIDERAÇÕES FINAIS}

O presente artigo buscou evidenciar a relevância do estabelecimento de diálogo entre dois domínios específicos da produção de conhecimento, a saber, a neuropsicologia infantil e a oncologia pediátrica. Para tanto, abordou duas das principais neoplasias que acometem a faixa pediátrica, a saber, a leucemia linfoide aguda e os tumores de fossa posterior.

No que diz respeito a estes grupos clínicos, além dos efeitos neurocognitivos decorrentes da própria condição patológica e do tratamento administrado, condições adicionais podem conferir maior vulnerabilidade ao desenvolvimento de sequelas neurocognitivas adversas (Duffner, 2010). O conhecimento acerca da influência destes fatores de risco e variáveis sugere que nem toda criança sofrerá o mesmo grau de morbidade cognitiva pós-tratamento. Algumas crianças são capazes de permanecer relativamente inócuas a tais efeitos, ao passo que outras podem sofrer declínios de grande impacto em suas habilidades intelectuais e cognitivas (Moore III, 2005).

A idade da criança ao diagnóstico consiste em uma variável de grande relevância neste processo, uma vez que a partir desta é possível estimar as etapas maturacionais em curso no SNC. Sabendo-se que determinadas estratégias terapêuticas acarretam maior morbidade neurocognitiva quando administradas em estágios maturacionais sensíveis, caracterizados por maior vulnerabilidade a lesões e à exposição de agentes neurotóxicos, a idade é um dos primeiros critérios a serem considerados quando se pretende propor um protocolo terapêutico (Moore III, 2005). Entretanto, o impacto exercido pela idade da criança ao diagnóstico irá depender intimamente da natureza da lesão cerebral, a qual varia segundo o diagnóstico e, consequentemente, o tratamento adotado, gerando impactos em direções opostas em crianças submetidas a tratamento de tumores cerebelares benignos, por um lado, e crianças com tumores malignos ou LLA submetidas à radioterapia.

A intensidade da terapia também vem sendo referida como importante variável a influenciar o impacto cognitivo do tratamento. De acordo com Moore III (2005), em alguns casos, a utilização de dosagens elevadas - tanto de quimioterapia como de radioterapia - pode ser fonte de disfunções cognitivas mais graves. Em geral, crianças com meduloblastoma, submetidas a maiores dosagens de radioterapia (aproximadamente 36Gy, seguidas de reforço em fossa posterior), apresentam efeitos cognitivos mais acentuados do que as crianças com LLA, submetidas a dosagens menores de radioterapia (12 a 18Gy). Em geral, a dosagem terapêutica é adequada ao grupo de risco no qual o paciente é classificado, considerando critérios clínicos e biológicos (Lofstad et al., 2009). Entrentanto, a busca pelo equilíbrio entre a eficácia terapêutica e a manutenção da toxicidade do tratamento em níveis mínimos, tem desafiado os pesquisadores, visando garantir aos sobreviventes, além da reabilitação clínica, qualidade de vida e bem-estar após a cura (Moore III, 2005).

A influência do tempo decorrido a partir do diagnóstico nos resultados cognitivos de crianças com tumores de fossa posterior e LLA vem sendo fortemente apontada por diversos estudos (Palmer et al., 2007; Cole \& Kamen, 2006; Palmer, Gajjar, Reddick et al., 2003; Mulhern et al., 2001; Palmer, Goloubeva, Reddick et al., 2001; Ris, Packer, Goldwein et al., 2001; Moleski, 2000). A expressão clínica dos padrões de disfunções sofre modificações ao longo do tempo após o tratamento, cuja manifestação guarda íntima relação com a natureza da lesão e com a conjuntura maturacional em que o SNC se encontra. A relevância desta variável reside no fato de que esta representa o curso empreendido pelos mecanismos de 
reorganização pós-lesão, e a forma como estes emergem enquanto subprodutos da complexa e dinâmica relação entre a natureza, a localização e a extensão dos danos, por um lado, e os processos maturacionais em curso e a inserção sócio-cultural da criança, por outro. Em crianças com tumores cerebelares benignos, como o astrocitoma, não há evidências de progressão nos danos cognitivos, tendo em vista que a lesão mecânica acarreta danos focais imediatos, rapidamente estabilizados após processo inicial de reorganização (Cantelmi et al., 2008; Rønning, Sundet, Tønnessen et al., 2005). Em crianças com tumores cerebelares malignos, como o meduloblastoma, e crianças com LLA submetidas à quimio e/ou radioterapia, os danos aparentam ser progressivos, tendo em vista que a substância branca - principal componente afetado por tais estratégias terapêuticas - apresenta lenta taxa de replicação, e os efeitos de sua lesão sobre o funcionamento cognitivo tornam-se gradativamente perceptíveis, notadamente através da progressiva redução da velocidade de processamento e da lentidão no processo de aquisição de novas aprendizagens (Campbell et al., 2007; Palmer et al., 2007; Cole \& Kamen, 2006; Maddrey et al., 2005; Stargatt et al., 2002).

Outro aspecto que tem atraído a atenção dos pesquisadores é o sexo da criança, no que concerne às diferenças encontradas no desempenho cognitivo de meninos e meninas após o tratamento. Embora não haja consenso entre pesquisas com crianças de tumores de fossa posterior, pesquisas com crianças com LLA demonstram frequentemente que as meninas apresentam desempenho inferior àquele obtido por meninos, no entanto, a causa para tais achados ainda é desconhecida (Butler \& Haser, 2006). Alguns estudos sugerem que a explicação para os resultados supracitados pode ser encontrada na interação de fatores hormonais com os fármacos utilizados, ou mesmo devido a peculiaridades concernentes à maturação cerebral nos diferentes gêneros podem estar implicadas em tal disparidade (Von der Weid et al., 2003). Tem sido demonstrado que o aumento de substância branca - diretamente envolvida com o processo de mielinização - durante a infância, tende a ser menor nas meninas do que nos meninos, o que poderia tornálas mais vulneráveis aos efeitos da irradiação craniana e aos agentes neurotóxicos da quimioterapia (Buizer et al., 2009; Peterson, 2008).
No que diz respeito às crianças com tumores de fossa posterior, outra importante variável a ser considerada no processo de estabelecimento de prognóstico neuropsicológico é a presença de hidrocefalia, complicação bastante frequente nestes casos, devido à obstrução do IV ventrículo e ao bloqueio do fluxo de líquor (Mulhern \& Palmer, 2003). A hidrocefalia atualmente é considerada forte fator de risco ao desenvolvimento de sequelas neurocognitivas adversas em tais crianças, podendo resultar em danos extensos e difusos ao tecido cortical e subcortical, notadamente a substância branca, prejudicando o processo de mielinização das fibras axonais (Duffner, 2010; Cantelmi, Schweizer \& Cusimano, 2008; Hardy, Bonner, Willard et al., 2008; Stargatt, Anderson \& Rosenfeld, 2002; Erickson, Baron \& Fantie, 2001).

A localização do tumor é uma importante variável neste processo, uma vez que já se reconhece a especialização funcional de determinadas áreas da região da fossa posterior, em se destacando a participação de regiões cerebelares em funções mentais complexas, como as funções executivas (Schamamann et al., 1998, como citado em Bugalho, Correa \& Viana-Baptista, 2006). Lesões cerebelares comumente acarretam quadros disfuncionais específicos, como a Síndrome da Fossa Posterior e a Síndrome CognitivoAfetiva do Cerebelo, caracterizados essencialmente por alterações de caráter frontal/pré-frontal, às quais pode subjazer a existência de conexões cerebelofrontais recíprocas e a participação de ambas as regiões em um amplo sistema funcional complexo, compartilhando fatores neuropsicológicos componentes das funções executivas. Além disso, mais especificamente, reconhece-se atualmente que lesões do hemisfério cerebelar direito predominantemente estão associadas a alterações das funções de linguagem e de memória verbal, enquanto lesões do hemisfério esquerdo cerebelar associam-se primordialmente a dificuldades na realização de tarefas não verbais e de memória visual, achados justificados pela natureza cruzada das vias cerebelares (Bugalho et al., 2006; Riva \& Giorgi, 2000).

Espera-se que o presente estudo contribua para o incremento de novas pesquisas brasileiras no domínio da oncologia pediátrica, possibilitando maior compreensão acerca dos impactos oriundos do câncer e seus tratamentos, respaldando projetos de intervenção que garantam o desenvolvimento do potencial de aprendi- 
zagem, contribuindo diretamente com o aumento da qualidade de vida dessas crianças e adolescentes.

\section{REFERÊNCIAS}

Anderson, F. S. \& Kunin-Batson, A. S. (2009). Neurocognitive Late Effects of Chemotherapy in Children: The Pas 10 Years of Research on Brain Structure and Function. Pediatric Blood \& Cancer, 52, 159-164.

Ardila, A. (1996). Towards a Cross-Cultural Neuropsychology. Journal of Social an Evolutionary Systems, 19(3), 237-248.

Boone, K. B., Victor, T. L., Wen, J., Razani, J. \&Pontón, M. (2007). The association between neuropsychological scores and ethnicity, language, and acculturation variables in a large patient population. Archives of Clinical Neuropsychology, 22, 355-365.

Brinkman, T.M., Reddick, W.E, Luxton, J, Glass, J.O, Sabin ND, Srivastava D.K, Robison L.L, Hudson, M.M, Krull, K.R. (2012). Cerebral white matter integrity and executive function in adult survivors of childhood medulloblastoma. Neuro Oncol. 14 Suppl 4: iv25-36.

Budisavljevic, S., Ramnan, N. (2012). Cognitive deficits from a cerebellar tumour: A historical case report from Luria's Laboratory. Cortex, 4 8, 2 6-3 5.

Bugalho, P., Correa, B., \& Viana-Baptista, M. (2006). Papel do cerebelo nas funções cognitivas e comportamentais: bases científicas e modelos de estudo. Acta Médica Portuguesa, 19, 257-268.

Buizer, A. I., Soneville, L. M. J. \& Veerman, A. J. P. (2009). Effects of Chemotherapy on Neurocognitive Function in Children With Acute Lymphoblastic Leukemia: A Critical Review of the Literature. Pediatric Blood Cancer, 52, 447-454.

Butler, R. W. \& Haser, J. K. (2006). Neurocognitive effects of treatment for childhood cancer. Mental Retardation and Developmental Disabilities Research Review, 12(3), 184-191.

Campbell, L. K., Scaduto, M., Sharp, W., Dufton, L., Van Slyke, D., Whitlock, J. A. \& Compas, B. (2007). A meta-analysis of the neurocognitive sequelae of treatment for childhood acute lymphocytic leukemia. Pediatric Blood and Cancer, 49(1), 65-73.

Cantelmi, D., Schweizer, T. A. \& Cusimano, M. D. (2008). Role of the cerebellum in the neurocognitive sequelae of treatment of tumours of the posterior fossa: an update. The Lancet Oncology, 9, 569-576.

Cole, P. D. \& Kamen, B. A. (2006). Delayed neurotoxicity associated with therapy for children with acute lymphoblastic leukemia. Mental Retardation and Developmental Disabilities Research Review, 12(3), 174-183.

Duffner, P. K. (2010). Risk factors for cognitive decline in children treated for brain tumors. European Journal of Paediatric Neurology, 14, 106-115.
Erickson, K., Baron, I. S. \&Fantie, B. D. (2001). Neuropsychological functioning in early hydrocephalus: review from a developmental perspective. ChildNeuropsychology, 7(4), 199-229.

Garcia, D. (2011). Investigação da capacidade intelectiva de pacientes pediátricos diagnosticados com tumores de fossa posterior. Dissertação de Mestrado, Programa de Pós-Graduação em Psicologia, Universidade Federal do Rio Grande do Norte, Natal.

Gomes, E. (2011). Investigação do funcionamento cognitivo em pacientes pediátricos diagnosticados com Leucemia Linfoide Aguda- LLA. Dissertação de Mestrado, Universidade Federal do Rio Grande do Norte, Natal

Hardy, K. K., Bonner, M. J., Willard, V. W., Watral, M. A. \& Gururangan, S. (2008). Hydrocephalus as a possible additional contributor to cognitive outcome in survivors of pediatric medulloblastoma. Psycho-Oncology, 17, $1157-1161$.

Hazin, I. Dellatolas, G., Garcia, D., Pedrosa, F. \& Pedrosa, A. (2011). Intellectual impairment after treatment for medulloblastoma and astrocytoma in childhood: the Brazilian experience. Journal of Pediatric Hematology Oncology, 33(7), 506-515.

Hazin, I., Leitão, S., Garcia, D., Gomes, E. \& Lemos, C. (2010). Contribuições da Neuropsicologia de Alexsander Romanovich Luria para o debate contemporâneo sobre relações mente-cérebro. Mnemosine,6(1), 88-110.

Law, N., Bouffet, E., Laughlin, S., Laperriere, N., Brière, M., Strother, D., McConnell, D., Hukin, J., Fryer, C., Rockel, C., Dickson, J., \& Mabbott, D. (2011). Cerebello-thalamo-cerebral connections in pediatric brain tumor patients: Impact on working memory. NeuroImage, 56, 2238-2248.

Maddrey, A. M., Bergeron, J. A., Lombardo, E. R., McDonald, N. K., Mulne, A. F., Barenberg, P. D. \& Bowers, D. C. (2005). Neuropsychological performance and quality of life of 10 year survivors of childhood meduloblastoma. Journal of Neuro-Oncology, 72, 245253.

Massimino, M., Cefalo, G., Riva, D., Biassoni, V., Spreafico, F., Pecori, E., Poggi, G., Collini, P., Pollo, B., Valentini, L., Potepan, P., Seregni, E., Casanova, M., Ferrari, A., Luksch, R., Polastri, D., Terenziani, M., Pallotti, F., Clerici, CA., Schiavello, E., Simonetti, F., Meazza, C., Catania, S., Podda, M., Gandola, L. (2012). Long-term results of combined preradiation chemotherapy and agetailored radiotherapy doses for childhood medulloblastoma. Journal of Neuro-Oncology, 108(1):163-71.

Mello, C. B., Miranda, M. C., Feldman, C., Sinnes, E. G., Barbosa, T., Beltrami, M. C. et al. (2006b). Abordagem interdisciplinar em lesões encefálicas na infância. In C. B. Mello, M. C. Miranda \& M. Muszkat (orgs.), Neuropsicologia do desenvolvimento: conceitos e abordagens (pp. 223-236). São Paulo: Memnon. 
Moleski, M. (2000). Neuropsychological, Neuroanatomical, and Neurophysiological Consequences of CNS Chemotherapy for Acute Lymphoblastic Leukemia. Archives of Clinical Neuropsychology, 15 (7),603-630.

Moore III, B. D. (2005). Neurocognitive Outcomes in Survivors of Childhood Cancer. Journal of Pediatric Psychology, 30(1), 51-63.

Mulhern, R. K. \& Butler, R. W. (2004). Neurocognitive sequelae of childhood cancers and their treatment. Pediatric Rehabilitation, 7(1), 1-14.

Mulhern, R. K., Palmer, S. L., Merchant, T. E., Wallace, D., Kocak, M., Brouwers, P. et al. (2005). Neurocognitive Consequences of Risk-Adapted Therapy for Childhood Medulloblastoma. Journal of Clinical Oncology, 23, 5511-5519.

Mulhern, R. K., Palmer, S. L., Reddick, W. E., Glass, J. O., Kun, L. E., Taylor, J. et al. (2001). Risks of young age for selective neurocognitive deficits in medulloblastoma are associated with white matter loss. Journal of Clinical Oncology, 19(2), 472-479.

Mulhern, R., \& Palmer, S. (2003). Neurocognitive late effects in pediatric cancer. Current Problems in Cancer, 27, 177-197.

Mulhern, R. K., Reddick, W. E., Palmer, S. L., Glass, J. O., Elkin, T.D., Kun, L. E., Taylor, J., Langston, J. \& Gajjar, A. (1999a). Neurocognitive deficits in Medulloblastoma are survivors and white matter loss. Annals of Neuro$\log y, 46,834-84$.

Muszkat, M. (2008c). Neurodesenvolvimento e neuroplasticidade. In M. Muszkat\& C. B. de Mello (orgs.), Neuropsicologia do Desenvolvimento e suas interfaces (pp. 51-72). São Paulo: All Print.

Noogle, C. \& Dean, R. (2013). The neuropsychology of cancer and oncology. New York: Springer.

Palmer, S. L., Gajjar, A., Reddick, W. E., Glass, J. O., Kun, L. E., Wu, S. et al. (2003). Predicting intellectual outcome among children treated with 35-40 Gycraniospinalirradiation for medulloblastoma. Neuropsychology, 17(4), 548-555.

Palmer, S. L., Goloubeva, O., Reddick, W. E., Glass, J. O., Gajjar, A., Kun, L. et al. (2001). Patterns of intellectual development among survivors of pediatric medulloblastoma: a longitudinal analysis. Journal of Clinical Oncology, 19(8), 2302-2308.

Palmer, S., Reddick, W. E. \& Gajjar.A. (2007). Understanding the cognitive impact of in children who are treated for medulloblastoma. Journal of Pediatric Psychology, 32(9), 1040-1049.

Peterson, C., Johnson, C. E., Ramirez, L. Y., Huestis, S., Pai, A. L. H., Demaree, H. A. \& Drotar, D. (2008). A meta-analysis of the neuropsychological sequelae of chemotherapy-only treatment for pediatric acute lym- phoblastic leukemia. Pediatric Blood Cancer, 51(1), 99104.

Raymond-Speden, E., Tripp, G., Lawrence, B. \& Holdaway, D. (2000). Intellectual, neuropsychological, and academic functioning in long-term survivors of leukemia. Journal of Pediatric Psychology, 25(2), 59-68.

Ris, M., D., Packer, R., Goldwein, J., Jones-Wallace, D. \& Boyett, J. M. (2001). Intellectual outcome after reduceddose radiation therapy plus adjuvant chemotherapy for medulloblastoma: a children's cancer group study. Journal of Clinical Oncology, 19 (15), 3470-3476.

Riva. D. \& Giorgi, C. (2000). The cerebellum contributes to higher functions during development: evidence from a series of children surgically treated for posterior fossa tumours. Brain, 123, 1051-1061.

Rønning, C., Sundet, K., Tønnessen, B. D., Lundara, T \& Helseth, E. (2005). Persistent cognitive dysfunction secondary to cerebellar injury in patients treated for posterior fossa tumors in childhood. Pediatric Neurosurgery, $41,15-21$.

Spiegler, J., Kennedy, K., Maze, R., Greenberg, M., Weitzman, S., Hitzler, J. \& Nathan, P. (2006). Comparison of Long-Term Neurocognitive Outcomes in Young Children with Acute Lymphoblastic Leukemia Treated with Cranial Radiation or High-Dose or Very High-Dose Intravenous Methotrexate. Journal of Clinical Oncology, 24 (24), 3858-3864.

Stargatt, R., Anderson, V. \& Rosenfeld, J. V. (2002). Neuropsychological outcomes of children treated for posterior fossa tumours: a review. Brain Impairment, 3(2), 92104.

Stargatt, R., Rosenfeld, J. V., Anderson, V., Hassall, T., Maixner, W. \& Ashley, D. (2006). Intelligence and adaptive function in children diagnosed with brain tumour during infancy. Journal of Neurooncology, 80, 295-303.

Temning, P. \& Jenney, M. E. M. (2010). The neurodevelopmental sequelae of childhood leukaemia and its treatment. Archives of Disease in Childhood, 95, 936-940.

Varela, M., Liakopoulou, M., Alexiou, G., Pitsouni, D., Alevizopoulos, G. (2011). Presurgical neuropsychological and behavioral evaluation of children with posterior fossa tumors. J. Neurosurg Pediatrics. (8), 548-553.

Watanabe, S., Azami, Y., Ozawa, M., Kamiya, T., Hasegawa, D., Ogawa, C., Ishida, Y., Hosoya, R., Kizu, J. \& Manabe, A. (2011). Intellectual development after treatment in children with acute leukemia and brain tumor. Pediatr Int 53(5), 694-700.

Recebido em: 14/01/2013

Revisões requeridas em: 12/12/2013 Aceito em: 23/08/2014 